\title{
The Direction-finding Stations Scheduling Algorithm Based on BPSO
}

\author{
Sun Tai-chuan ${ }^{1, a}$, Zhang Jing $^{1, b}$, Ran Xiao-min $^{1, \mathrm{c}}$, LI Yao-dong ${ }^{1, \mathrm{~d}}$ \\ ${ }^{1}$ I.T.Institute of Zhengzhou, Henan Province, China \\ a aSwjtu20092258@126.com, b'zhangjingok@sina.com, \\ c13298181526@163.com, ${ }^{\mathrm{d}} 511242140 @ q q . c o m$
}

\begin{abstract}
Keywords: Fisher information matrix, Binary Particle Swarm Optimization, finding co-location, cyclic
\end{abstract} shift initialization;

\begin{abstract}
To solve the scheduling problems of stations in cooperative localization, binary particle swarm algorithm of scheduling direction-finding stations is proposed based on Fisher information matrix(FIM).This paper makes the trace of FIM matrix as positioning accuracy measure,constructs scheduling model that contains positioning accuracy, signal to noise ratio(SNR) and task balanced three optimization objectives. In the process of solving the model,this paper uses the particle self-cyclic shift initialization method and checking the constraint of fitness function to improve the Binary Particle Swarm Optimization(BPSO).Simulation results show that optimizing the track of FIM can improve the positioning accuracy,compared with the penalty function method, the fitness function by constraint checking can greatly reduce the computational complexity and the improved BPSO convergences faster.
\end{abstract}

\section{Introduction}

In recent years, as the rapid development of anti-radiation missiles and electronic jamming technology, active positioning is threatened more and more seriously,while passive positioning technology has good concealment, strong anti-interference ability,long detection distance , resistance to destroy, etc.In particular, it plays a very important role in terms of covert detection and precision strike,leading the increasing attention around the world.According to the coordination number,passive positioning can be divided single station positioning and multi-station location.Compared with the single station location, multi-station location has the advantages of high accuracy, high speed, full orientation, etc.and plays an important role in the passive location.Factors affecting the multi-station positioning accuracy for many reasons, includes the localization algorithm, filtering algorithm, disposition form,etc.At present, our cooperative direction positioning scheduling rely mainly on artificial scheduling.After decades of development, the limitations of artificial scheduling is gradually revealed, the problems they face primarily is the task of heavy, inefficient, signal leakage test, uneven distribution and so on.Since direction-finding station measured signal intensity band, signal more, especially when a large number of concurrent signals, artificial scheduling is almost impossible to complete the task.Resource utilization rate is not high, also causes other signal missing,the low task completion rate.Therefore, an efficient intelligent direction-finding station scheduling algorithm becomes more and more necessary for the direction-finding stations scheduling.

The current research on direction-finding station scheduling problem, haven't found in the open Reference.Through analysis, its essence is a combination of multi-objective optimization problem, we can learn traditional research scheduling problem to solve the problem.This paper mainly researches the FIM and BPSO to solve the direction-finding station scheduling problem.In many countries,FIM has been widely used in various fields.In [1] FIM is applyed in statistical data model of poisson image reconstruction method to improve the resolution of consistency.Reference [2] uses the FIM and virtual noise of covariance function to optimize parameters.Reference [3] specializes in monte carlo calculation of FIM standard parameter is set, proposing a resampling-based method to calculate the relevant information of FIM.Ben-Haim and Eldar [4] introduced the FIM constraints of Cramér-Rao low bound(CRLB), and the experimental results shows that the CRLB does not exist in 
unbiased estimation,but under the condition of biased estimation can be constructed.Reference [5] describes the prospects for the use of FIM in gravitational wave parameter estimation. BPSO in recent years on a very wide application issued a swarm intelligence algorithm.Reference [6] using the BPSO configuration observability power system power management units. Reference [7] divided the particle velocity into different levels, and proposed a new rule to update and improve the convergence speed of the particle, and successfully applied to gene selection to obtain better results.

\section{Scheduling Model Based on Information Matrix}

\section{A. Measurement Model and Assumptions}

In this paper,the tracking problem for $N_{r}$ targets is considered in 2-D field .According to general measurement model in Reference [20], which is expressed as :

$$
z_{i, k}=\left(1+\omega_{i, k}\right) r_{i, k}+v_{i, k}
$$

where $z_{i, k}$ stands for the measurement of direction-finding station $i . \omega_{i, k}$ and $v_{i, k}$ be uncorrelated white Gaussian noise,i.e., $\omega_{i, k} \sim N\left(\mu_{\omega}, \sigma_{\omega}^{2}\right), v_{i, k} \sim N\left(\mu_{v}, \sigma_{v}^{2}\right)$, where

$$
\begin{aligned}
& z_{i, k} \sim N\left(\mu_{i, k}+r_{i, k}, \sigma_{i, k}^{2}\right) \\
& \mu_{i, k}=r_{i, k} \mu_{\omega}+\mu_{v} \\
& \sigma_{i, k}^{2}=r_{i, k}^{2} \sigma_{\omega}^{2}+\sigma_{v}^{2}
\end{aligned}
$$

Arbitrary direction finding the distance between the station and the target signal can be expressed as:

$$
r_{i, k}=\sqrt{\left(x_{i}-x_{k}\right)^{2}+\left(y_{i}-y_{k}\right)^{2}}
$$

Where $s_{i}=\left(x_{i}, y_{i}\right)$ stands for the coordinates of direction-finding station $i, t_{k}=\left(x_{k}, y_{k}\right)$ stands for the target prediction coordinates of direction-finding station $k$.

The model to make the following assumptions:

1)The direction-finding station errors are independent of each other and fixed,where the mean $e_{i}$, and the variance $\sigma_{i}^{2}$.

2)Each direction-finding station that can independently to deal with data have all other direction-finding station fixed information,such as location,mean and variance.

3)The direction-finding stations can communicate each other.

4)The signal-to-noise ratio requirements for each direction-finding stations is different.There is a fixed threshold $S n r_{0}$, When $S n r_{j} \geq S n r_{0}$, the signal can be effective direction finding, when $S n r_{j}<S n r_{0}$, will not be effective for signal direction finding.

Defines $A$ direction-finding station selection matrix, $A=\left[a_{j, k}\right]_{N_{s} \times N_{r}}, a_{j, k}$ stand for the choice of relations between said direction-finding station and task, where

$$
a_{j, k}= \begin{cases}1 & \text { finding } \\ 0 & \text { else }\end{cases}
$$

\section{B. Model index}

Tracking precision.This paper uses the trace of FIM matrix as indicators of positioning accuracy.FIM matrix, as the application of probability of mutual information,is from the perspective of probability of edge information to each other to gaining maximization of mutual information from the matrix.In this way,the information of matrix can provide more contribution for positioning accuracy.The FIM of target signal proposed in [9] as follows:

$$
\boldsymbol{I}_{k}=\sum_{i=1}^{M} g_{i, k}\left[\begin{array}{cc}
\cos ^{2} \varphi_{i, k} & \cos \phi_{k, i} \sin \varphi_{i, k} \\
\cos \varphi_{i, k} \sin \phi_{i, k} & \sin ^{2} \varphi_{i, k}
\end{array}\right]
$$




$$
g_{i, k}=\frac{1}{\sigma_{i, k}^{4}}\left[\sigma_{i, k}^{2}\left(1+\mu_{\omega}\right)^{2}+2 \sigma_{\omega}^{4} r_{i, k}^{2}\right]
$$

Where $\cos \varphi_{i, k}=\left(x_{i}-x_{k}\right) / r_{i, k}$, and $\sin \varphi_{i, k}=\left(y_{i}-y_{k}\right) / r_{i, k}$ 。By the formula (10) shows that, FIM is a matrix, can not be directly calculated and compared.Reference [10] it has demonstrated that maximizing the trace of FIM matrix and maximizing matrix are equivalent.Therefore, this paper will trace FIM as finding stations scheduled for an optimization goal.By equation (10) can be obtained:

$$
D_{k}=\operatorname{det}\left\{I_{k}\right\}=\frac{1}{2} \sum_{i=1}^{N_{z}} \sum_{j=1}^{N_{r}} g_{i, k} g_{j, k} \sin ^{2}\left(\varphi_{i j, k}\right)
$$

In order to facilitate with other optimization goal in the same order of magnitude, this paper will divide the trace of FIM matrix $D_{k}$ by its average $D_{k, a v e}$.

$$
f_{1}=\frac{D_{k}}{D_{k, a v e}}
$$

The sum of signal-to-noise ratio Signal to noise ratio target signals measured will be another optimization objective in this paper.Through the Sigmoid function, map it to [0,1].In order to ensure that each measure scheduled to have practical effect, set a threshold, the threshold value greater than or equal to as a scheduling objects, or do not assign a scheduled task.

$$
b_{j, k}= \begin{cases}1 & \operatorname{Snr}_{i}(k) \geq S n r_{0} \\ 0 & \text { else }\end{cases}
$$

In order to facilitate with other optimization goal in the same order of magnitude, SNR is dealt with Sigmoid function and values mapped to $[0,1]$ interval, namely

$$
f_{2}=\left\{\begin{array}{cc}
\frac{1}{M N_{r}} \sum_{i=1}^{N_{z}} \sum_{k=1}^{M} \frac{4}{e^{-\frac{S n r_{i}(k)-S n r_{i, \text { min }}}{S n r_{i, \max }-S n r_{i, \text { min }}}}+1}-2 & S n r_{i, \text { max }}-S n r_{i, \text { min }} \neq 0 \\
0 & \text { else }
\end{array}\right.
$$

Where $M$ stand for the numbers of direction-finding stations with collaborative direction positioning, $S n r_{i}(k)$ stand for the signal-to-noise ratio of target signal scheduled, $S n r_{i, \max }$ 、 $S n r_{i, \min }$ respectively stand for the maximum and minimum values for each station of the target signal to noise ratio forecast.

Task equilibrium degree.In order to select the station with less task. This paper defines a busy degree $B(i)$,scheduled times and value is mapped to the interval $[0,1]$ by Sigmoid Function.

$$
B(i)=\left\{\begin{array}{cc}
3-\frac{4}{-\frac{B s y(i)-B s y_{\min }}{B s y_{\max }-B s y_{\min }}}+1 & B s y_{\max }-B s y_{\min } \neq 0 \\
0 & \text { else }
\end{array}\right.
$$

Where Bsy(i) stand for the current scheduled times of direction-finding station $i$.respectively stand for the maximum and the minimum number of direction-finding stations have been scheduled.

$$
f_{3}=\frac{1}{N_{r}} \sum_{i=1}^{N_{r}} B(i)
$$

\section{Scheduling Algorithm Based on The BPSO}

PSO (Particle Swarm Optimizer, PSO) is evolutionary algorithm by simulating the natural phenomena.as well as Genetic Algorithm[11], Ant Colony Algorithm[12], Greedy Algorithm[13] .It was originally developed by Eberhart and Kennedy [14] in 1995 based on the behavior of birds of prey presented.But when handling of 0-1 problem, PSO efficiency is low, therefore, in 1997 in view of the problem of discrete BPSO is designed in [15].Based on the study of BPSO (pso) algorithm, this paper combines the actual conditions of direction-finding station scheduling problem, by using the vector encoding, particles from cyclic shift initialization and conversion function to improve the BPSO. 


\section{A. Initialization Method}

First to reduce the search space particles,generation and the constraint conditions that meet the needs of the particles, through the SNR threshold $S n r_{0}$ and direction-finding station coordination number $X_{t}$, sparse direction-finding station selection matrix $A=\left[a_{j, k}\right]_{N_{s} \times N_{r}}$.

Then by reference [16] sensor scheduling problem in the research put forward a kind of cyclic shift initialization (cyclic shift initiation, CSI), this paper proposes a Particle self-cyclic shift initiation(PSCSI).

\begin{tabular}{|l|c|c|c|c|c|c|c|c|}
\hline 0 & 1 & $\ldots$ & 1 & $\ldots$ & 1 & 0 & $\cdots$ & 1 \\
\hline 1 & 0 & $\cdots$ & 1 & $\ldots$ & 1 & 1 & $\ldots$ & 0 \\
\hline
\end{tabular}

Fig.1 The processing of self-cyclic shift initiation

\section{B. Particle Velocity and Position Update}

In the basic BPSO, each particle represents a feasible solution, the position of the particles determines the size of the fitness function value ,also decides the pros and cons.Each particle in the process of optimization, based on their current location, the global best position and local best position status updates. The equation of the particle's velocity and position update is as follow ${ }^{[15]}$.

$$
\begin{gathered}
v_{i d}^{l+1}=w \cdot v_{i d}^{l}+c_{1} \cdot \operatorname{rand}() \cdot\left(p_{\text {best }, \text { id }}^{l}-x_{i d}^{l}\right)+c_{2} \cdot \operatorname{rand}() \cdot\left(\mathrm{g}_{\text {best }, i d}^{l}-x_{i d}^{l}\right) \\
x_{i d}^{l+1}=\left\{\begin{array}{l}
1, \text { if } \operatorname{rand}() \leq S\left(v_{i d}^{l+1}\right) \\
0, \text { if } \operatorname{rand}()>S\left(v_{i d}^{l+1}\right)
\end{array}\right. \\
S\left(v_{i d}^{l+1}\right)=1 /\left(1+\exp \left(-v_{i d}^{l+1}\right)\right)
\end{gathered}
$$

Reference [17] has proved V-shaped conversion function compared with S-shaped transition function, has faster convergence speed and better avoids falling into local optimal solution at the same time.

$$
T\left(v_{i d}^{l+1}\right)=\left|\operatorname{erf}\left(\frac{\sqrt{\pi}}{2}\right) v_{i d}^{l+1}\right|=\left|\frac{\sqrt{2}}{\pi} \int_{0}^{\frac{\sqrt{\pi}}{2} v_{i d}^{l+1}} e^{-l^{2}} d l\right|
$$

\section{The Fitness Function}

Compared with the general multi-objective combinatorial optimization problem, direction-finding station scheduling problem, due to join the direction finding precision and signal-to-noise ratio, the respect such as task equilibrium calculation, making the computation of fitness function to occupy a large proportion in the whole optimization calculation.Checking the constraint of the fitness function,amount of calculation will reduce,because it needn't calculating the fitness function that doesn't satisfy the constraint conditions. Finally, using the linear weighting method to construct the fitness function.According to the target signal maneuverability is strong and not strong two cases, this paper puts forward two kinds of the design of fitness function.

1)fitness function by the the constraint checking.Maneuverable target signals need a fitness function with low complexity,that can quickly obtain the scheduling results. In the direction-finding station selection matrix $A=\left[a_{j, k}\right]_{N_{s} \times N_{r}}, X_{k}=\sum_{i=1}^{N_{z}} a_{j, k}$ stands for the number of stations of target $k$.

$$
f=\left\{\begin{array}{l}
\omega_{1} f_{1}+\omega_{2} f_{2}+\omega_{3} f_{3}, \text { if } X_{k}=X_{t} \quad \forall k \\
\infty, \text { if } X_{k} \neq X_{t} \quad \forall k
\end{array}\right.
$$

2)Fitness function using the penalty function method.In a large number of experiments, we found that some of the target signals scheduling process, when the the number of co-develop collaborative number is smaller than the constraint, the same effect can be achieved.Define a penalty factor $P u b$,constrained problem can be converted into unconstrained problem by penalty function method. 


$$
f=\omega_{1} f_{1}+\omega_{2} f_{2}+\omega_{3} f+\sum_{i=1}^{N_{r}} P u b \cdot\left(X_{k}-X_{t}\right)^{2}
$$

\section{The Results of Simulation and Analysis}

\section{A. The Simulation Environment Settings}

All experiments were performed herein is the use of $2.3 \mathrm{GHz}$ frequency of the PC for simulation in the matlab environment.Specific parameters are as follows: $S n r_{0}=1, R_{t} \leq 100 \mathrm{~km}, \omega_{1}=0.5, \omega_{2}=0.3, \omega_{3}=0.2, \mu_{\omega}=\mu_{v}=0, \sigma_{v}^{2}=\sigma_{\omega}^{2}=1$.In the process of each experiment, the input of the number and position of target signal and direction finding are randomly generated.In order to verify the validity of the proposed algorithm, this paper uses the monte carlo method.

\section{B. Simulation Results Analysis}

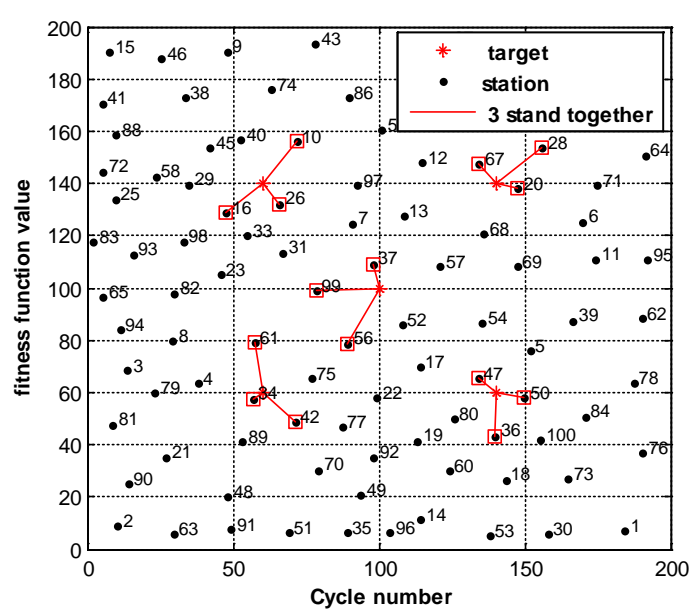

Fig.2The results of scheduling

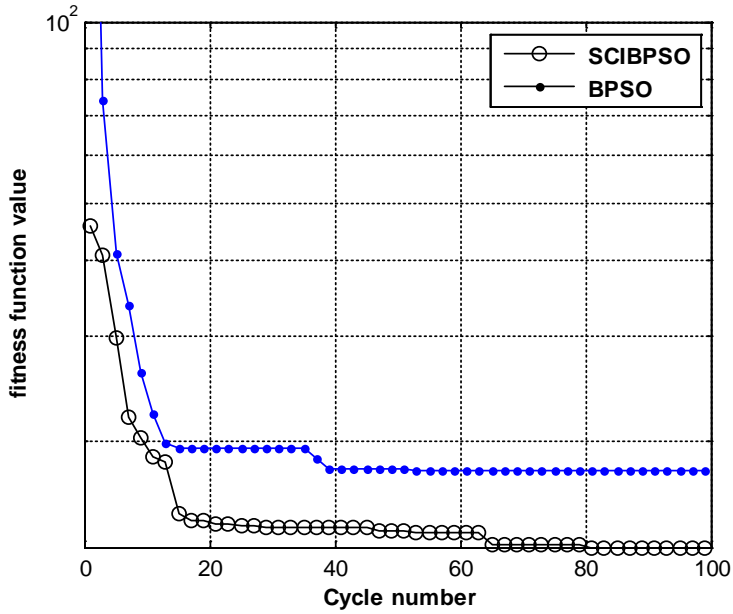

Fig.3 The contrast SCIBPSO and BPSO

Figure 2 shows the results five target signals with three stations to co-locate scheduling.As can be seen from the figure 2, the proposed algorithm can effectively solve the problems. The convergent processing of contrast between BPSO and the algorithm this paper proposed is in figure 3.As can be seen from Figure 3, the proposed algorithm converges is faster and the solution is better.

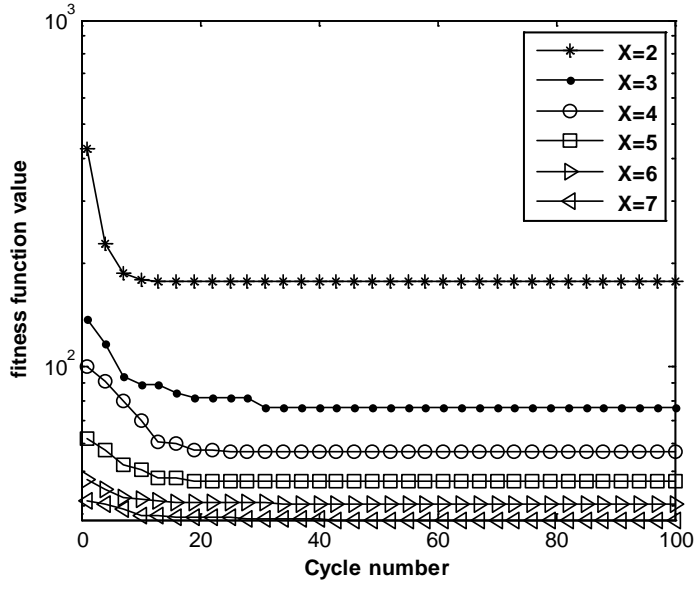

Fig. 4 The results of different coordination number

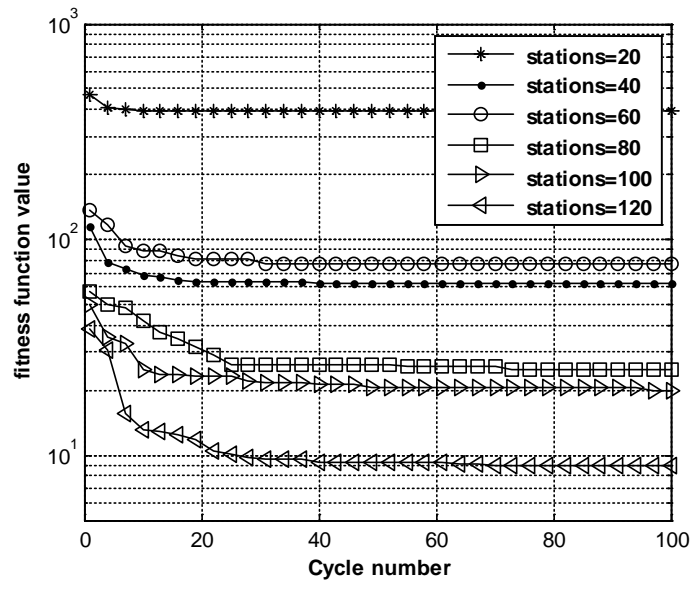

Fig.5 The results of different stations

Figure 4 is convergence process diagram with the different collaborative numbers.As can be seen from the figure 4, with the increase of coordination number, the desires are better under the same cycles.Taken together, the best synergy number is 5 .Figure 5 is convergence process diagram with the different direction-finding stations.As can be seen from the figure 4,the more stations, and the solution better.The best numbers of direction-finding stations is 80 . 


\section{Conclusion}

This paper mainly study the scheduling model and algorithm.Improving particle initialization method and introducing the V-shaped conversion function improved the convergence of BPSO.Introducing the trace of FIM as positioning accuracy can be effectively applied scheduling problems of direction-finding stations.Through simulation experiments, we can obtain the optimum number of coordination and station.

\section{References}

[1] Li Q, Asma E, Qi J, et al. Accurate estimation of the FIM matrix for the PET image reconstruction problem[C]// IEEE Trans. Med. Imag. 2004:1057 - 1064.

[2] Pazman A. Correlated optimum design with parametrized covariance function. Justification of the FIM matrix and of the method of virtual noise[J]. 2004.

[3] Spall J C. Monte Carlo Computation of the FIM Matrix in Nonstandard Settings[J]. Taylor \& Francis, 2012, 14(4):889-909.

[4] Ben-Haim Z, Eldar Y C. On the Constrained CramÉr-Rao Bound With a Singular FIM Matrix[J]. Signal Processing Letters IEEE, 2009, 16(6):453 - 456.

[5] Vallisneri M. Use and abuse of the FIM matrix in the assessment of gravitational-wave parameter-estimation prospects[J]. Phys.rev.d, 2008, 77(4):-.

[6] Chakrabarti S, Venayagamoorthy G K, Kyriakides E. PMU placement for power system observability using BPSO[C]// Power Engineering Conference, 2008. AUPEC '08. Australasian Universities. IEEE, 2009:1-5.

[7] Mohd Saberi M, Sigeru O, Safaai D, et al. A modified BPSO for selecting the small subset of informative genes from gene expression data.[J]. IEEE Transactions on Information Technology in Biomedicine A Publication of the IEEE Engineering in Medicine \& Biology Society, 2011, 15(6):813-822.

[8] Wang $\mathrm{X}, \mathrm{Fu} \mathrm{M}$, Zhang $\mathrm{H}$. Target Tracking in Wireless Sensor Networks Based on the Combination of KF and MLE Using Distance Measurements[J]. Mobile Computing IEEE Transactions on, 2012, 11(4):567-576.

[9] Yang Z Y, Shi X F, Chen J M. Optimal Coordination of Mobile Sensors for Target Tracking Under Additive and Multiplicative Noises [J]. IEEE Transactions on Industrial Electronics, 2014, 61(7):3459-3468.

[10]Bishopk A N, Jensfelt P. An Optimality Analysis of Sensor-Target Geometries for Signal Strength Based Localization [C] //Proceedings of the 5th ISSNIP, VIC December 7-10, 2009:127-132.

[11]Deb K, Pratap A, Agarwal S, et al. A fast and elitist multi-objective genetic algorithm: NSGA II[J]. IEEE Transactions on Evolutionary Computation, 2002, 6(2):182-197.

[12]Maier H R, Simpson A R, Foong K, et al. Ant Colony Optimization for the Design of Water Distribution Systems[J]. American Society of Civil Engineers, 2014, (2001):1-10.

[13]Bocu R, Tabirca S. The Flag-based Algorithm - A Novel Greedy Method that Optimizes Protein Communities Detection[J]. International Journal of Computers Communications \& Control, 2011, 6(1):33-44.

[14]Eberhart R, Kennedy J. A new optimizer using particle swarm theory[C]// Micro Machine and Human Science, 1995. MHS '95., Proceedings of the Sixth International Symposium on. IEEE, 1995:39-43.

[15]Kennedy J, Eberhart R C. A discrete binary version of the particle swarm algorithm[C]// Systems, Man, and Cybernetics, 1997. Computational Cybernetics and Simulation., 1997 IEEE International Conference on. IEEE, 1997:4104-4108 vol.5.

[16]Naeem M, Pareek U, Lee D C. Swarm Intelligence for Sensor Selection Problems[J]. IEEE Sensors Journal, 2012, 12(8):2577-2585.

[17]Mirjalili S, Lewis A. S-shaped versus V-shaped transfer functions for BPSO[J]. Swarm \& Evolutionary Computation, 2013, 9:1-14. 